\title{
Clinical and Radiologic Analysis of Disc Heights Change in Conservatively Treated Lumbar Herniated Disc Patients
}

\author{
Dong-Min Seo, Yongjae Cho, Na-Re Yang \\ Department of Neurosurgery, Ewha Women's University Mokdong Hospital, Ewha Womans University College of Medicine,
} Seoul, Republic of Korea

Corresponding author:

Yongjae Cho

Objective: The main purpose of this study was to evaluate the clinical and radiologic factors of disc

Department of Neurosurgery, Ewha Methods: From January 2017 to December 2018, a total of 56 symptomatic herniated lumbar disc Women's University Mokdong Hospital, Ewha Womans University College of Medicine, 1071, Anyangcheon-ro, Yangcheon-gu, Seoul 07985, Republic of Korea Tel: $+82-2-2650-2685$

Fax: +82-2-2650-2651

E-mail: yongcho@ewha.ac.kr

Received: April 6, 2021

Revised: April 30, 2021

Accepted: May 24, 2021 patients (36 men and 20 women) were investigated retrospectively in this study. Clinical findings including age, sex, pain, and smoking history were examined in each group (group A, patients with not-changed disc heights; group B, patients with decreased disc heights). Radiologically, the level, severity, laterality, modic change, and disc degeneration of lumbar disc herniation were investigated between groups. We compared demographic and radiologic findings between groups.

Results: A total of 56 patients were enrolled (group A, 30 patients; group B, 26 patients). There were no significant statistical differences in age, sex, visual analog scale (VAS) scores, level of disc herniation, and laterality of disc herniation between groups. Group A had more protrusion cases (14/30 cases) and group B had more Sequestration (11/26 cases). About Modic change and disc degeneration classification, group B had more modic change cases and more disc degeneration cases than group B. Conclusion: In this study, there were no significant differences in demographic findings, level of disc herniation, the status of disc herniation, laterality of disc herniation, VAS of back pain between group $A$ and group B.

Key Words: Conservative treatment; Intervertebral disc disease; Lumbar vertebrae

\section{INTRODUCTION}

Lumbar disc hemiation, which causes low back pain and radiating pain in the lower extremities, is a common disease in 20\% to 30\% of the population ${ }^{6}$. Since Mixter and Barr ${ }^{8}$ introduced surgical treatment for lumbar disc herniation in 1934, discectomy has been performed as a standard treatment for this disease. However, it has been reported that the lumbar disc herniation-induced low back pain and radiating pain in the lower extremities improves spontaneously without surgical treatment. In 1984, Guinto et al. ${ }^{2}$ reported for the first time that the herniated intervertebral disc disappeared spontaneously through computed tomography imaging. Following this, numerous cases of spontaneous recovery have been published. This has been the basis for conducting conservative treatment for lumbar disc herniation ${ }^{10}$. Disc space height was decreased after discectomy due to surgical reduction of disc material, which has been widely known. But, few studies have assessed changes in disc height in patients who are treated conservatively.

In this study, we retrospectively analyzed patients whose herniated lumbar disc disappeared spontaneously after conservative treatment and assessed the change in disc space height. The main purpose of this study was to evaluate the clinical and radiologic factors of disc height loss in spontaneous resolution of herniated lumbar disc patients. Demographic, clinical, and radiologic findings such as the level, status, severity, Modic change, and disc degeneration of lumbar disc herniation were assessed.

\section{MATERIALS AND METHODS}

\section{Patients}

We retrospectively analyzed 56 symptomatic herniated lumbar disc patients from 226 patients diagnosed with an acute symptomatic herniated lumbar disc from January 2017 to December 2018 in our department. Fifty-six patients (36 men and 20 women) were retrospectively chosen for this study. Patients selection criteria included: (1) $<50$ years of age with buttocks and leg pain concomitantly associated with a herniated lumbar disc; (2) one level of hermiated lumbar disc (protrusion, extrusion, and sequestration) diagnosed on lumbar magnetic resonance (MR) imaging (MRI); (3) conservative treatment choice (medication and physical therapy) after medical explanation; and (4) regular hospital visits for 2 years with lumbar MRI during follow up after initial MRI. The exclusion criteria were as follows (1) spine trauma history; (2) history of cancer; (3) previous lumbar surgery; and (4) a change in treatment options, such as interventional block and surgery. Disc heights (initial and last follow- up MRI) were measured at T2 sagittal MR using modified 
method from Farfan ${ }^{4)}$ (Fig. 1). For evaluation of disc endplates and disc degeneration, Modic changes and Pfirmann grading was used, respectively (T) $^{5}$ (Table 1, 2).

We analyzed and organized two patient groups according to the difference between initial and last measured disc height. Group A included a patient with no or $<2 \mathrm{~mm}$ disc height (Hereinafter referred to as not-changed disc height group). Group B included patients with $\geq 2 \mathrm{~mm}$ decrease in disc height (Hereinafter referred to as decreased disc height group).

Lumbar MRI was performed twice during this study with a 3.0 Tesla imager (Philips Medical Systems, Best, Netherlands) from T10

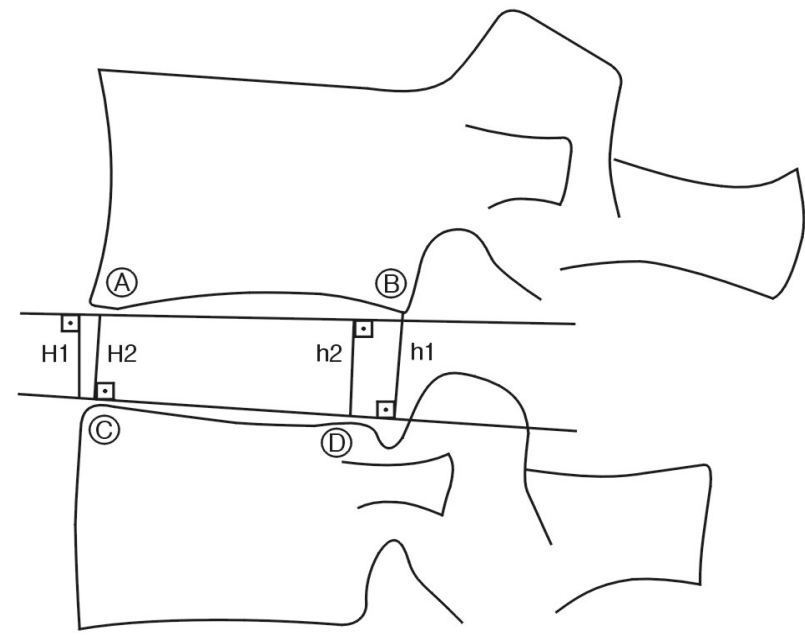

Fig. 1. Measurement of the intervertebral disc height according to the modified Farfan method. Disc height $=(\mathrm{H} 1+\mathrm{H} 2+\mathrm{h} 1+\mathrm{h} 2) / 4$. (A): Anteroinferior corner of the superior vertebra, B posteroinferior corner of the superior vertebra; (C) anterosuperior corner of the inferior vertebra, (D) posterosuperior corner of the inferior vertebra. Adapted from reference 4 . to S5. The MR protocol was adjusted by a radiologist. All images were uploaded to the in-hospital picture archive and communication system (PACS; Infinitt PACS, invented by Infinitt Co., Seoul, Korea) and reviewed using the workstation-base unit (Hewlett-Packard Co., Palo Alto, CA, USA). All 112 MR images of 56 patients were reviewed independently by two experts (one neurosurgeon and one radiologist) who had not been given any information about the subjects. Lumbar vertebrae were counted from S1. The status of disc hemiation was defined as below: Protrusion of disc hemiation is focal hemiation which has a broader base than the body, while extrusion has a narrower base than the body. When there is already continuity loss with the disc itself, it is called disc sequestration.

\section{Analysis}

Clinical characteristics, including age, sex, smoking history and visual analog scale (VAS; 10 points for back and leg) score were examined in each group. Radiologically, the level, status, laterality of lumbar disc herniation, Modic change, and disc degeneration grade were investigated between groups. Clinical and radiologic factors were compared to determine those that caused height differences between groups (Table 3).

The clinical and radiologic analysis was performed using the SPSS statistical package version 22.0 software (IBM Corp., Armonk, NY, USA) using an independent samples t-test and $\chi^{2}$ test. Statistical significance was measured to be when the $p$-value was less than 0.05. The study was approved by the Institutional Review Board (IRB) of the Ewha Womans Mokdong Hospital Bioethics Committee (IRB No. 2020-06-16-0017).

\section{RESULTS}

Patients ages ranged from 18 to 49 years (mean, $32.4 \pm 11.3$ years in group A, 35.8 \pm 13.3 in group B) (Table 3). The total group

Table 1. Endplate changes using Modic classification

\begin{tabular}{cccl}
\hline \hline Type & T1-weighted images & T2-weighted images & \multicolumn{1}{c}{ Description } \\
\hline I & Low signal & High signal & Edema and inflammation of bone marrow \\
II & High signal & high signal & Marrow ischemia; yellow fatty marrow; transformation \\
III & Low signal & Low signal & Sclerosis over subchondral bony area \\
\hline
\end{tabular}

Table 2. Disc degeneration classification using Pfirrmann grading

\begin{tabular}{|c|c|c|c|c|}
\hline Grade & Structure & Distinction nucleus and annulus & Signal intensity & Height of intervertebral disc \\
\hline 1 & Homogenous, bright white & Clear & $\begin{array}{l}\text { Hyperintense, isointense to } \\
\text { cerebrospinal fluid }\end{array}$ & Normal \\
\hline$\|$ & $\begin{array}{l}\text { In homogenous with or } \\
\text { without horizontal bands }\end{array}$ & Clear & $\begin{array}{l}\text { Hyperintense, isointense to } \\
\text { cerebrospinal fluid }\end{array}$ & Normal \\
\hline III & Inhomogenous, gray & Unclear & Intermediate & Normal to slightly decreased \\
\hline IV & Inhomogenous, gray to black & Lost & Intermediate to hypointense & Normal to moderately decreased \\
\hline V & Inhomogenous, black & Lost & Hypointense & Collapsed disc space \\
\hline
\end{tabular}


mean age was 38.4 years and the male to female ratio was 1.8:1 (36 males and 20 females). The initial VAS scores in group A and $B$ were 4.8 and 4.7 points, respectively. The VAS scores at the time of last follow-up were 2.4 and 2.3 points in group $A$ and B. There were no significant differences in age, sex ratio, smoking ratio, and VAS scores between groups.

The mean differences (initial disc height-last disc height) were $0.88 \pm 0.75 \mathrm{~mm}$ and $2.16 \pm 0.64$ in group $A$ and $B$, respectively, which was significant $(p=0.021)$. However, patients were divided into two groups according to disc height differences (Table 3). Initial mean disc heights were $12.15 \pm 0.15 \mathrm{~mm}$ and $12.48 \pm 0.35$ in group
$A$ and $B$, respectively, which were not different between the two groups in disc height at the initial diagnosis. The last mean of disc heights was $11.89 \pm 0.41 \mathrm{~mm}$ in group $A$ and $10.23 \pm 0.82$ in group B. The initial disc height was no difference between the two groups, but the last disc height of group B was less than group A (Table 3).

Level of disc herniation was observed in group A: 5 cases in $L 3 / 4,17$ in $L 4 / 5$, and 8 in L5/S1 and in group B: 4 cases in L3/ 4,21 in $L 4 / 5$, and 7 in $L 5 / S 1$. In both groups, disc herniation was the most common in L4/5, followed by LS/S1 and L3/4. Status of disc hemiation revealed, there were 14 cases protrusion, 7 extrusion, and 9 sequestration in group A. There were 5 cases protrusion, 10

Table 3. Demographical and radiologic data of herniated lumbar disc patients

\begin{tabular}{|c|c|c|c|}
\hline & Group $A^{\ddagger}(n=30)$ & Group $B^{\S}(n=26)$ & p-value \\
\hline Sex (Male:Female) & 18:12 & $18: 8$ & \\
\hline Mean age & $32.4 \pm 11.3$ & $35.8 \pm 13.3$ & 0.072 \\
\hline Smoking & $14(46.7 \%)$ & $12(46.2 \%)$ & \\
\hline \multicolumn{4}{|l|}{ VAS (back pain) † } \\
\hline Initial & 4.8 & 4.7 & 0.452 \\
\hline Follow-up & 2.4 & 2.3 & 0.448 \\
\hline \multicolumn{4}{|l|}{ Disc height } \\
\hline Initial & $12.15 \pm 0.15$ & $12.48 \pm 0.35$ & 0.132 \\
\hline Follow-up & $11.89 \pm 0.41$ & $10.23 \pm 0.82$ & $0.034^{*}$ \\
\hline Difference (initial - follow-up) & $0.88 \pm 0.75$ & $2.16 \pm 0.64$ & $0.021^{*}$ \\
\hline \multicolumn{4}{|l|}{ Modic change } \\
\hline Normal & $24(80.0 \%)$ & 13 (50.0\%) & $0.025^{*}$ \\
\hline Grade 1 & $3(10.0 \%)$ & $8(30.8 \%)$ & 0.091 \\
\hline Grade 2 & $2(6.67 \%)$ & $4(15.4 \%)$ & 0.083 \\
\hline Grade 3 & 1 (3.33\%) & $1(3.85 \%)$ & 0.082 \\
\hline \multicolumn{4}{|l|}{ Disc degeneration classification } \\
\hline Grade 1 & $14(46.7 \%)$ & $0(0.0 \%)$ & \\
\hline Grade 2 & $16(53.3 \%)$ & $0(0.0 \%)$ & \\
\hline Grade 3 & $0(0.0 \%)$ & $8(30.8 \%)$ & \\
\hline Grade 4 & $0(0.0 \%)$ & 18 (69.2\%) & \\
\hline Grade 5 & $0(0.0 \%)$ & $0(0.0 \%)$ & \\
\hline \multicolumn{4}{|l|}{ Level of disc herniation } \\
\hline $\mathrm{L} 3 / 4$ & $5(16.7 \%)$ & $4(15.4 \%)$ & 0.167 \\
\hline$L 4 / 5$ & $17(56.7 \%)$ & 21 (80.8\%) & 0.075 \\
\hline$L 5 / S 1$ & $8(26.7 \%)$ & 7 (26.9\%) & 0.132 \\
\hline \multicolumn{4}{|l|}{ Status of disc herniation } \\
\hline Protrusion & $14(46.7 \%)$ & $5(19.2 \%)$ & $0.036^{*}$ \\
\hline Extrusion & 7 (23.3\%) & 10 (38.5\%) & 0.082 \\
\hline Sequestration & $9(30.0 \%)$ & $11(42.3 \%)$ & 0.175 \\
\hline \multicolumn{4}{|l|}{ Laterality of disc herniation } \\
\hline Left:Right & 13:17 & 14:12 & \\
\hline
\end{tabular}

The data is presented as number (\%) or mean \pm standard deviation.

VAS: visual analog scale.

${ }^{*} p<0.05 .{ }^{+}$VAS (10 points). ${ }^{\dagger}$ Group A: patients with not-changed disc height. ${ }^{\S}$ Group B: patients with decreased disc heights. 


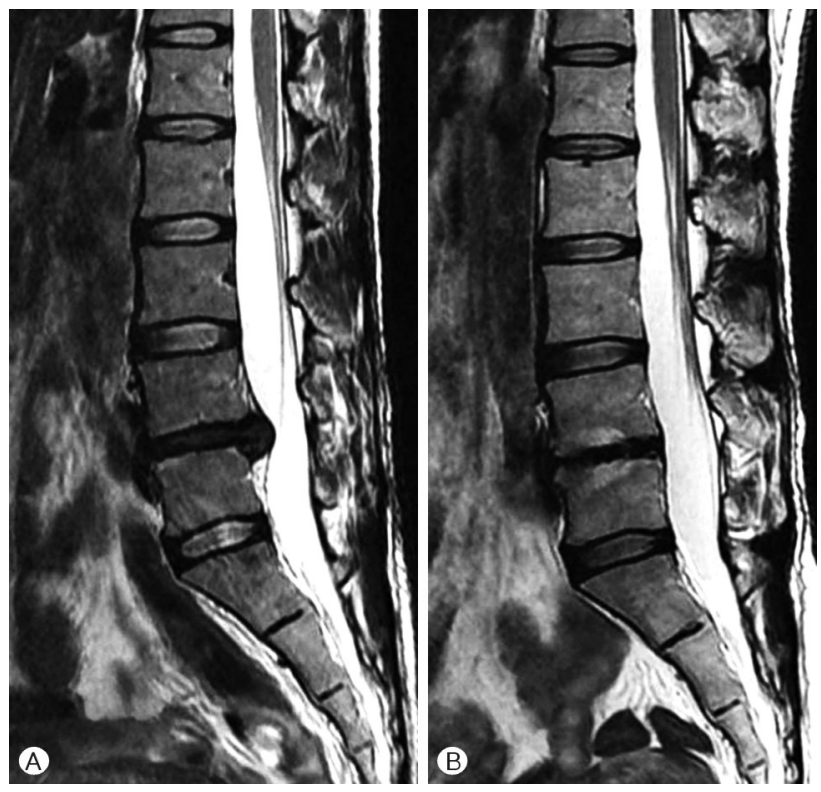

Fig. 2. A 32-year-old man with symptomatic lumbar disc herniation. He suffered from mild back pain and left leg pain for 3 weeeks. Magnetic resonance (MR) findings. (A) Initial MR imaging (MRI), showing a large area of disc extrusion at the $L 4 / 5$ disc space. (B) MRI taken 1 year after the initial MRl, showing spontaneous regression of the herniated lumbar disc and loss of disc height.

extrusion, and 11 sequestration in group $B$. There were more protrusion cases (14/30) in group A than group B. In contrast, extrusion and sequestration were more common in group $B(10 / 26$ and 11/ 26 cases, respectively. The ratio of laterality of disc herniation (left: right) were $13: 17$ and 14:12 in group $A$ and $B$, respectively. There were no significant statistical differences in level of disc herniation and laterality of disc herniation between groups (Table 3).

About Modic change, group A had more normal cases than group B: 24 cases (80\%) in the not-changed disc height group, 13 cases (50\%) in decreased disc height group. According to disc degenera- tion classification, all cases of not-changed disc height group belon- ged to both grade 1 and grade 2, and all cases of decreased disc height group belonged to grade 3,4 , and 5 .

\section{DISCUSSION}

Lumbar disc herniation has been known to occur predominantly in $L 3 / 4, L 4 / 5$, and $L 5 / S 1$ and can be further classified into protrusion, extrusion, and sequestration. Disc protrusion was demarcated as a focal or asymmetric extension of the disc beyond the vertebral border, with the protruded origin wider than any other aspect of the protrusion. Extrusion was defined as a more extreme extension of the disc beyond the vertebral border, with the base against the disc of origin slenderer than the diameter of the extruding material and a connection between the material and the disc of origin. Sequestration was defined as a free disc fragment that was distinct from the origin disc.
In this study, we divided patients treated conservatively into two groups according to their change in disc heights (Fig. 2).

It was observed that the decreased disc height patients group had more extrusion and sequestration cases than not-changed disc height patients group (Table 3). These results suggested two things: first, by morphology of disc herniation, extrusion and sequestration are expected to have more herniated disc when compared with protrusion. Secondly, extrusion and sequestration are likely to have a ruptured posterior longitudinal ligament (PLL) compared with protrusion. In general, it can be said that the higher the amount of herniation and the more the PLL supporting posterior aspect of disc ruptures, the higher the possibility that the disc height will be lowered later.

Several pathophysiological mechanisms have been reported for the natural regression of the herniated intervertebral disc; however, the exact mechanism has not been elucidated ${ }^{7}$. Three hypotheses have been proposed in the literature. First, the prolapsed intervertebral disc returns to the intervertebral disc space by a healthy $\mathrm{PLL}^{13)}$. Second, the size of the intervertebral disc is reduced and resolved by gradual dehydration and contraction, Third, the herniated intervertebral disc causes immunologic, biochemical responses and neovascularization, which results in phagocytosis and enzymatic degradation of cartilage tissue ${ }^{12,13)}$. Among them, the most recently noted hypothesis of spontaneous resolution is that intervertebral disc tissue is absorbed by immune responses and neovascularization, which be inferred from the rim enhancement of end plate of disc space on the Gadolinium-enhanced MRI in patients with a lumbar herniated $\operatorname{disc}^{3,13)}$

In this study, decreased disc height patients group had more Modic change and disc degeneration than not-changed disc height patients group (Table 3). This suggested that more degenerative changes cause disc height to decrease later.

Surgical treatment is considered when there is persistent pain or neurological symptoms despite the proper period of conservative treatment. Surgery creates a dramatic recovery of symptoms in lumbar intervertebral disc herniation; however, the incidence of recurrence or complications after surgery is reported to be $5 \%$ to $10 \%$ ') Additionally, there have been reports that removal of a protruded lumbar intervertebral disc may accelerate degenerative changes in the intervertebral disc and end plates, resulting in a loss of disc space height, Modic change, and post-discectomy back pain ").

In this study, spontaneously resolved disc herniation without discectomy showed a decrease in disc height at long-term follow-up MRI. Conservative treatment is an effective treatment for herniated lumbar disc; however, it is necessary to recognize lumbar intervertebral disc herniation as a risk factor that can cause the loss of intervertebral disc height along with disc degeneration and Modic changes $5,9,11$ ).

There were no differences in back pain between not-changed disc height patients and decreased disc height patients. To date, the relationship between disc height reduction or disc degeneration and back pain remains unclear ${ }^{5,11}$ ). In addition to discussing with patients the options of conservative treatment for herniated lumbar disc, it is necessary to explain that herniated lumbar disc itself can cause 
a decrease in disc height and chronic back pain due to degenerative changes in the intervertebral disc.

Further research is required on the factors that are associated with degenerative changes in lumbar intervertebral disc herniation.

This study has some limitations. The main disadvantage is the retrospective nature. We organized the patient groups and selected patients into groups, which creates a bias. Additionally, the number of patients in this study was small and the follow-up periods were relatively short to obtain significant conclusions. Therefore, further research with larger patient populations is required to fully elucidate the underlying mechanisms and assess radiological and clinical evaluations.

\section{CONCLUSION}

In conservative treatment option for symptomatic lumbar disc herniation, a decrease of disc height may occur later and it would be more at the cases of extrusion and sequestration type herniation. This disc height decrease after lumbar disc hemiation without surgical removal of disc may be due to disc degeneration and disc hemiation itself. It is necessary to explain to patients who are treated conservatively that disc height may be reduced later.

\section{CONFLICTS OF INTEREST}

No potential conflict of interest relevant to this article was reported.

\section{REFERENCES}

1. Barth M, Diepers M, Weiss C, Thomé C: Two-year outcome after lumbar microdiscectomy versus microscopic sequestrectomy: part 2: radiographic evaluation and correlation with clinical outcome. Spine (Phila Pa 1976) 33:273-279, 2008

2. Guinto FC, Jr., Hashim H, Stumer M: CT demonstration of disk regression after conservative therapy. AJNR Am J Neuroradiol 5:632-633, 1984
3. Henmi T, Sairyo K, Nakano S, Kanematsu Y, Kajikawa T, Katoh S, et al.: Natural history of extruded lumbar intervertebral disc herniation. J Med Invest 49:40-43, 2002

4. Hong $\mathrm{CH}$, Park JS, Jung KJ, Kim WJ: Measurement of the normal lumbar intervertebral disc space using magnetic resonance imaging. Asian Spine J 4:1-6, 2010

5. Jensen TS, Karppinen J, Sorensen JS, Niinimäki J, LeboeufYde C: Vertebral endplate signal changes (Modic change): A systematic literature review of prevalence and association with non-specific low back pain. Eur Spine J 17:1407-1422, 2008

6. Kim DK, Oh CH, Lee MS, Yoon SH, Park HC, Park CO: Prevalence of lumbar disc herniation in adolescent males in Seoul, Korea: Prevalence of adolescent LDH in Seoul, Korea. Korean J Spine 8:261-266, 2011

7. Komori H, Shinomiya K, Nakai O, Yamaura I, Takeda S, Furuya K: The natural history of herniated nucleus pulposus with radiculopathy. Spine (Phila Pa 1976) 21:225-229, 1996

8. Mixter WJ, Barr JS: Rupture of the intervertebral disc with involvement of the spinal canal. N Engl J Med 211:210-215, 1934

9. Ohtori S, Yamashita M, Yamauchi K, Inoue G, Koshi T, Suzuki $\mathrm{M}$, et al.: Low back pain after lumbar discectomy in patients showing endplate modic type 1 change. Spine (Phila Pa 1976) 35:E596-E600, 2010

10. Postacchini F: Lumbar disc herniation: a new equilibrium is needed between nonoperative and operative treatment. Spine (Phila Pa 1976) 26:601, 2001

11. Shahmohammadi MR, Behrouzian S: Effect of preoperative modic change in the outcome of patients with low back pain following posterior spinal fusion or laminectomy. Asian J Neurosurg 14:432-435, 2019

12. Slavin KV, Raja A, Thornton J, Wagner FC, Jr.: Spontaneous regression of a large lumbar disc herniation: Report of an illustrative case. Surg Neurol 56:333-337, 2001

13. Turk O, Antar V, Yaldiz C: Spontaneous regression of herniated nucleus pulposus: The clinical findings of 76 patients. Medicine (Baltimore) 98:e14667, 2019 